\title{
Self-organized two-dimensional onions
}

\author{
Shenqiang Ren, Robert M. Briber, and Manfred Wuttig ${ }^{\text {a) }}$ \\ Materials Science and Engineering, University of Maryland, College Park, Maryland 20742-2115, USA
}

(Received 27 January 2009; accepted 26 February 2009; published online xx xx xxxx)

\begin{abstract}
Spontaneously self-assembled onion-type nanostructures based on block copolymers as templating materials are reported. Polystyrene-poly(ethylene oxide) diblock copolymer containing $\mathrm{CoFe}_{2} \mathrm{O}_{4}$ and $\mathrm{Pb}_{1.1}\left(\mathrm{Zr}_{0.53} \mathrm{Ti}_{0.47}\right) \mathrm{O}_{3}$ precursors segregated to the two microdomains forms well-ordered templated lamellar structures. Onion-type nanostructures have been induced by room temperature solvent annealing for $64 \mathrm{~h}$ in a magnetic field of $0.8 \mathrm{~T}$ oriented perpendicularly to the plane of film. The recorded images suggest that the Lorentz force acting on charges in the paraelectric precursor induces a circular component of the diffusion flux that leads to the onion formation. This templating process opens a route for nanometer-scale patterning of magnetic toroids. () 2009 American Institute of Physics. [DOI: 10.1063/1.3101373]
\end{abstract}

Block copolymers have attracted much attention as prominent systems for nanotechnological applications because of their ability to spontaneously develop patterns on a nanoscale based on self-assembly. ${ }^{1-4}$ Currently, a great deal of attention is being paid to the synthesis of complex nanostructured inorganic-organic hybrid materials. ${ }^{5-10}$ A typical approach is the use of organic structures formed through self-assembly as structure directing agents. The final morphology is then determined by the cooperative organization of inorganic and organic molecular species into threedimensionally structured arrays. ${ }^{11}$ In thin films, in addition to composition and molecular weight, the domain structure is also dependent on the surface energies of the blocks and on geometrical constraints introduced by the film. A recent example of inorganic-organic hybrid templating is the fabrication of ultrahigh density ferromagnetic nanocylinders that are embedded in a piezoelectric matrix by utilizing a precursorcontaining polystyrene-block-polyethylene oxide block copolymer. ${ }^{12}$ The morphology and orientations of microdomains are the key factors controlling the properties of the patterned materials. The limited control over order and orientation of the microdomain in thin films poses restrictions on possible usages of patterning innovative material configurations via block copolymers. ${ }^{13}$

Recent work demonstrates the alignment of block copolymers by electromagnetic fields. ${ }^{14,15}$ Electric field induced alignment has been shown to be especially useful in thin film systems in which the two blocks have different dielectric constants. It does, however, require special sample geometries and surface preparation in the form of electrodes and patterned surfaces. Ultimately, dielectric breakdown limits the applicability of electric fields to alter block copolymeric patterns. ${ }^{16-18}$ Magnetic fields should in principle also lead to spatial reorientations of copolymer blocks. They are attractive as no limitations to the field strength and sample configuration exist. Magnetic field induced alignment was reported in $1998 ;{ }^{19}$ a $2.4 \mathrm{~T}$ magnetic field was imposed on a diblock copolymer comprising both block chains of liquidcrystalline polymers, resulting in a weak orientation order. Similar alignment has also been demonstrated for semicrystalline polymers in the presence of a strong magnetic field of

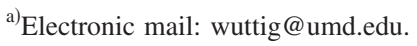

$30 \mathrm{~T}^{20-23}$ Due to the small interaction energies magnetic reorientation requires immense fields that are technologically unrealistic. Here we demonstrate that low magnetic field annealing of a directed assembly of a homogeneous diblock copolymer with multiferroic $\mathrm{Pb}_{1.1}\left(\mathrm{Zr}_{0.53} \mathrm{Ti}_{0.47}\right) \mathrm{O}_{3}(\mathrm{PZT})$ and $\mathrm{CoFe}_{2} \mathrm{O}_{4}$ (CFO) precursors produces a well-developed selforganized two-dimensional (2D) "onion" nanostructure. This organization is accomplished by a Lorentz force acting on a permanent charge present in the PZT portion.

Sol-gel PZT and CFO precursors were synthesized by mixed metal organic polymerization. The PZT precursor was prepared by adding acetylacetone to a mixture of titanium isopropoxide and zirconium propoxide in a 1:1 molar ratio with respect to the (Ti/Zr) alkoxides. To this mixture lead acetate trihydrate, diluted with 1,3-propanediol at a 1:5 molar ratio, was added. The final solution was dissolved into propylene glycol monomethyl ether. The CFO precursor consisted of a solution containing cobalt 2-methoxyethoxide and iron acetylacetonate metal alkoxide in 2-methoxyethanol. The CFO and PZT precursors were subsequently added sequentially to a commercially available polystyrene-blockpoly(ethylene oxide) (PS- $b$-PEO) (molecular weight of $9500 \mathrm{~g} \mathrm{~mol}^{-1}$; polydispersity of 1.07) with a PEO volume fraction of 0.6 (Polymer Sources, Inc.) copolymer dissolved in tetrahydrofuran (THF, $10 \mathrm{mg} \mathrm{ml}^{-1}$ ). Films were spun from this PZT/CFO precursor doped diblock copolymer solution onto a $\mathrm{SiO}_{2} / \mathrm{Si}$ substrate at $3000 \mathrm{rpm}$ for a period of $20 \mathrm{~s}$ and subsequently solvent (chloroform) annealed at room temperature for different times in a magnetic field of different strengths directed perpendicularly to the film surface. Rings with up to 46 layers, a period of $15 \mathrm{~nm}$ and diameters as large as $1.5 \mu \mathrm{m}$, can be seen. A transmission electron microscopy (TEM) image of the resulting domain structure is shown in Fig. 1. Low magnification TEM plane-view images demonstrate that the onions are aperiodically arranged and occupy approximately $85 \%$ of the surface area. Energy dispersive $\mathrm{x}$-ray spectrometer spectra obtained from the bright and dark rings of the film confirm the chemical composition of the separated blocks. In this state and at room temperature the domains are superparamagnetic and strongly paraelectric, respectively.

The magnetic field induced morphological evolution of the PS(PZT)- $b$-PEO(CFO) thin films was examined by 


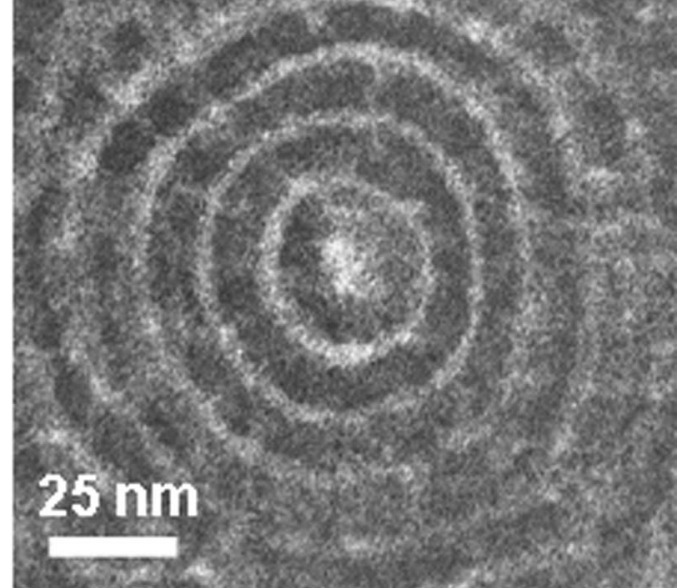

FIG. 1. TEM cross section of the 2D onion domain structure created by annealing a PS/PZT- $b$-PEO/CFO polymer for $\approx 3840 \mathrm{~min}(64 \mathrm{~h})$ in a magnetic field of $0.8 \mathrm{~T}$. The PZT and CFO precursors serve as contrasting agents.

atomic force microscopy (AFM). Figure 2 shows a series of AFM phase-contrast images taken after increasing times of anneals in magnetic fields of increasing strengths. They reveal that onion-type morphologies form at magnetic fields of 0.8 T. Auxiliary experiments had established that this morphology does not evolve if the PS block of the diblock copolymer was not doped with PZT. This fact will be the starting point of the discussion below. Fast Fourier transforms (FFTs) corresponding to the sequence of AFM images in Fig. 2, displayed in Fig. 3, demonstrate the conceptual evolution of the $2 \mathrm{D}$ onion morphology: the known laminar pattern evolves if the anneal is conducted without a magnetic field; at small magnetic fields lamellar order predominates; at intermediate fields a modulated 2D lamellar structure appears, while upon further increasing the magnetic field to $0.8 \mathrm{~T}$ the 2D onion structure develops.

The kinetics of the 2D onion evolution has been analyzed using GWYDDION software, ${ }^{24}$ which determines the av-

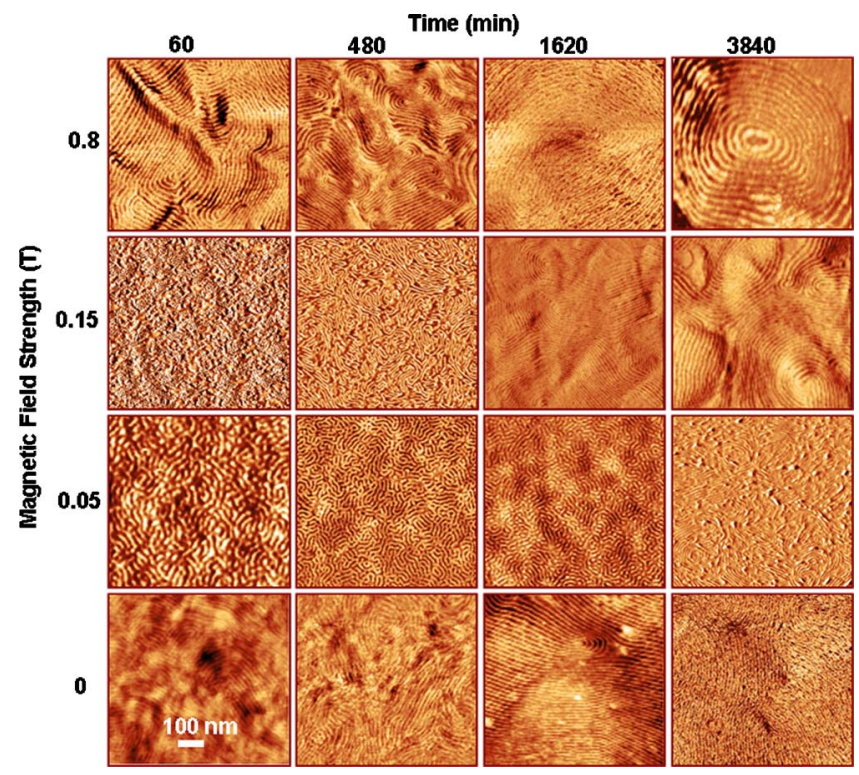

FIG. 2. (Color online) AFM phase images of the nanostructures created by room temperature annealing of a PS/PZT- $b$-PEO/CFO polymer for times as long as $\approx 3840 \mathrm{~min}(64 \mathrm{~h})$ in magnetic fields as high as $0.8 \mathrm{~T}$.

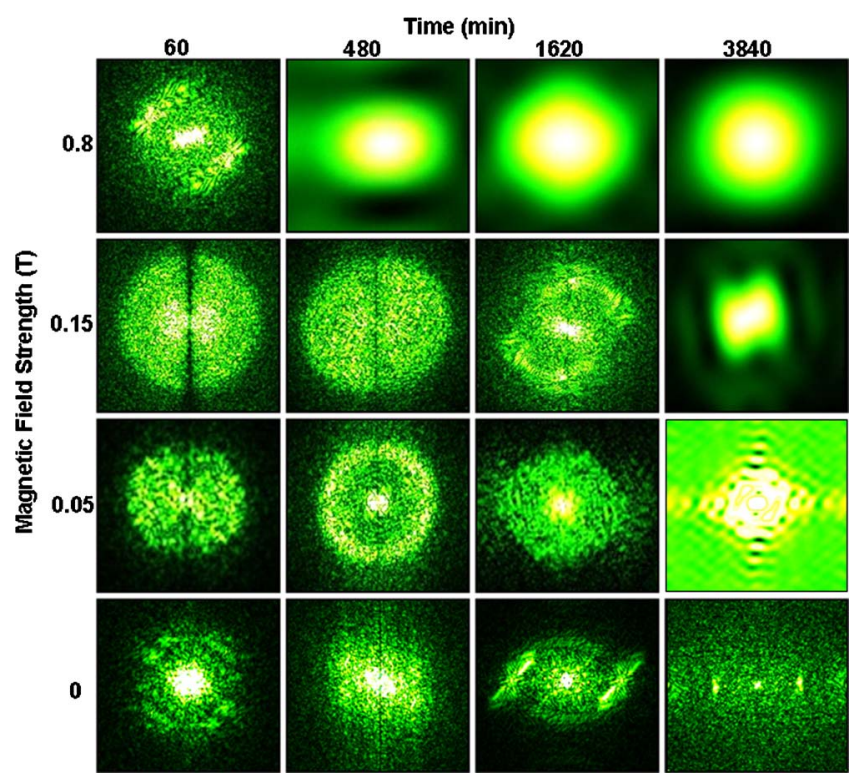

FIG. 3. (Color online) FFTs corresponding to the sequence of AFM images shown in Fig. 2.

erage curvature of the lines seen in the AFM nanostructures. Figure 4 presents the raw and normalized data. The analysis leading to the normalization is based on the observation that the paraelectric precursor, likely an associated electric charge, is required for the 2D onion-type formation, which points to a Lorentz force driven organization mechanism. This force is given by $\mathbf{F}=q[\nabla \mathbf{A} \times \mathbf{v}]$, where the three variables $q, \mathbf{A}$, and $\mathbf{v}$ denote the charge(s) in the PZT precursor, the magnetic vector potential due to the applied field, and the thermodynamic diffusion velocity of the polymer. This driving force can be included with the Cahn-Hilliard equation describing the evolution of the lamellar order ${ }^{25,26}$ by adding
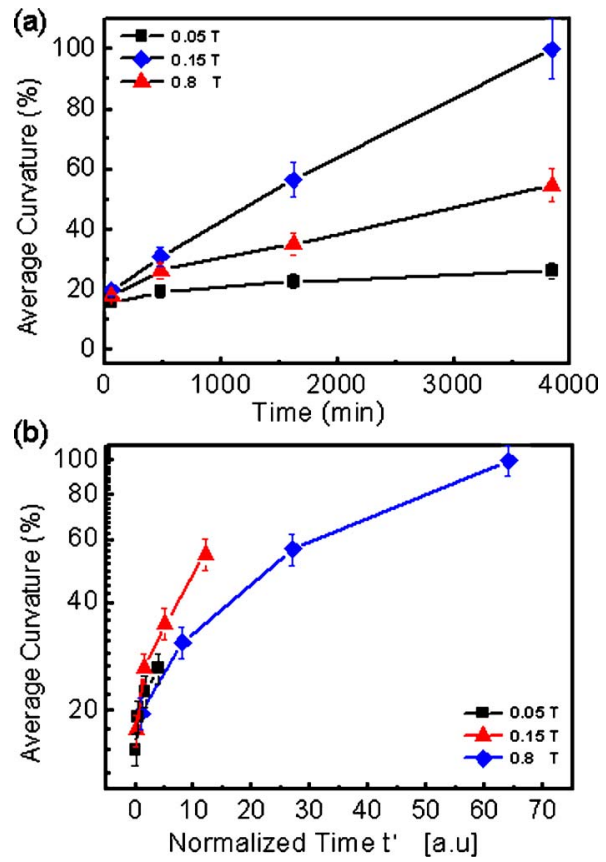

FIG. 4. (Color online) Initial growth kinetics of the $2 \mathrm{D}$ onion evolution in a $\mathrm{PS} / \mathrm{PZT}-b-\mathrm{PEO} / \mathrm{CFO}$ polymer thin film in the presence of a magnetic field $B$. Top: raw data; bottom: data normalized according to Eq. (2). Black squares, $B_{1}=0.05 \mathrm{~T}$; red triangles, $B_{2}=0.015 \mathrm{~T}$ : and blue diamonds, $B_{3}$ $=0.8 \mathrm{~T}$. 
the Lorentz term that drives the curvature. The linearized equation that can model the initial evolution of the observed $2 \mathrm{D}$ onion structure is then

$$
\Phi_{t}=M\left[\left(g_{\Phi \Phi}-q B\right) \nabla^{2} \Phi-K \nabla^{4} \Phi\right], \quad g_{\Phi \Phi}<0 .
$$

The symbols $M, g_{\Phi \Phi}$, and $K$ denote the polymer chain mobility, second derivative of the free energy, and gradient energy, respectively. The maximal radial growth velocity with the wave vector $k_{r, m}^{2} \propto q B / K$ leads to the characteristic time of the initial exponential evolution of the curvature $\tau_{r, m}$ $\propto 1 / k_{r, m}^{2} D, D=M k T$-diffusivity. The kinetic raw data shown in Fig. 4 (top) can therefore be normalized as $t^{\prime}=t \cdot B^{(i)} / B^{(j)}$, where the indices of the flux densities refer to the data sets indicated in the caption of this figure. It can be seen from Fig. 4 (bottom) that the initial exponential growth of the curvature does normalize approximately in that fashion in agreement with the assertion that the Lorentz force induces the $2 \mathrm{D}$ onion structure. Further growth must be described by the full nonlinear extension of the analysis. The FFTs indicate that for small fields $B$, lamellar order predominates, but an increasing Lorentz force modulates the emerging lamellae as signaled by the FFT in Fig. 3, taken after an anneal of $3840 \mathrm{~min}$ at $0.05 \mathrm{~T}$. For large magnetic fields the kinetics will approach the overdamped steady state motion of a charge in a magnetic field. Circular loops are formed, which, in the presence of a persistent lamellar order, become concentric cylinders separated by the interlamellar distance. We have observed similar diffusion enhanced dislocation free self-organization of plane-parallel lamellae in electric fields.

The onion-type nanostructures presented here may be technologically useful. Similar materials, termed mesoporous multilamellar vesicles, have been the synthetic target of numerous investigations because the structures could serve as efficient catalyst supports, adsorbents, or controlled-release materials for drug delivery. ${ }^{27-29}$ Warren et al. ${ }^{30}$ were able to synthesize $\mathrm{Au}$ core/silica shell nanoparticles by tailoring their size distributions. For most applications, it is desirable that the vesicle core and shell have different compositions, which is usually difficult to achieve. The present approach opens a simple pathway to the design of such compositionally heterogeneous structures.

In this paper we introduced directional diffusion control by external fields as a way to direct self-organization in precursor doped diblock polymers. The mechanism was demonstrated by the spontaneous assembly of $2 \mathrm{D}$ onions from $\mathrm{PS} / \mathrm{PZT}-b$-PEO/CFO polymer in a magnetic field. It is enabled by a charge in the PS/PZT block of this polymer. The two components, magnetic field and charge, combine to a
Lorentz force that creates the nanostructure. We have also observed the equivalent electric field enhancement of planeparallel nanostructures synthesized from the same doped diblock copolymer.

This work was supported by the grants and contracts from NSF (Grant No. DMR0705368), ONR-MURI (Grant No. N000140110761), ARO-MURI (Grant No. 28D1083899), and UMD-MRSEC program for permission to use the equipment.

${ }^{1}$ I. W. Hamley, Block Copolymers (Oxford Science, Oxford, 1999).

${ }^{2}$ M. P. Stoykovich and P. F. Nealey, Mater. Today 9, 20 (2006).

${ }^{3}$ C. J. Hawker and T. P. Russell, MRS Bull. 30, 952 (2005).

${ }^{4}$ R. A. Segalman, Mater. Sci. Eng. R. 48, 191 (2005).

${ }^{5}$ R. F. Service, Science 309, 95 (2005).

${ }^{6} \mathrm{H}$. Colfen and S. Mann, Angew. Chem., Int. Ed. 42, 2350 (2003).

${ }^{7}$ G. M. Whitesides, J. P. Mathias, and C. T. Seto, Science 254, 1312 (1991).

${ }^{8}$ G. M. Whitesides and B. Grzybowski, Science 295, 2418 (2002).

${ }^{9}$ C. T. Kresge, M. E. Leonowicz, W. J. Roth, J. C. Vartuli, and J. S. Beck, Nature (London) 359, 710 (1992).

${ }^{10}$ Y. Yin and A. P. Alivisatos, Nature (London) 437, 664 (2005).

${ }^{11}$ K. Schmidt, H. Schoberth, M. Ruppel, H. Zettl, H. Ansel, T. Weiss, V. Urban, G. Krausch, and A. Oker, Nature (London) 7, 142 (2008).

${ }^{12}$ S. Ren, R. Briber, and M. Wuttig, Appl. Phys. Lett. 93, 170507 (2008).

${ }^{13}$ P. G. D. Gennes and J. Prost, The Physics of Liquid Crystals, 2nd ed. (Clarendon, Oxford University Press, Oxford, New York, 1993), p. 597.

${ }^{14}$ A. Firouzi, D. J. Schaefer, S. H. Tolbert, G. D. Stucky, and B. F. Chmelka, J. Am. Chem. Soc. 119, 9466 (1997).

${ }^{15}$ Y. Tao, H. Zohar, B. Olsen, and R. Segalman, Nano Lett. 7, 2742 (2007).

${ }^{16} \mathrm{~K}$. Amundson, E. Helfand, X. Quan, and S. D. Smith, Macromolecules 26, 2698 (1993).

${ }^{17}$ T. L. Morkved, M. Lu, A. M. Urbas, E. Ehrichs, H. M. Jaeger, P. Mansky, and T. P. Russell, Science 273, 931 (1996).

${ }^{18}$ T. Thurn-Albrecht, J. Schotter, G. Kästle, N. Emley, T. Shibauchi, L. Krusin-Elbaum, K. Guarini, C. Black, M. Tuominen, and T. P. Russell, Science 290, 2126 (2000).

${ }^{19}$ D. Ferri, D. Wolff, J. Springer, O. Francescangeli, M. Laus, A. Angeloni, et al., J. Polym. Sci., Part B: Polym. Phys. 36, 21 (1998).

${ }^{20}$ H. Ezure, T. Kimura, S. Ogawa, and E. Ito, Macromolecules 30, 3600 (1997).

${ }^{21}$ T. Kimura, T. Kawai, and Y. Sakamoto, Polymer 41, 809 (2000).

${ }^{22}$ S. Sakurai, Polymer 49, 2781 (2008).

${ }^{23}$ T. Grigorova, S. Pispas, N. Hadjichristidis, and T. Thurn-Albrecht, Macromolecules 38, 7430 (2005).

${ }^{24}$ C. M. I. Inc. (available at http://www.gwyddion.net/).

${ }^{25}$ F. Liu and N. Goldenfeld, Phys. Rev. A 39, 4805 (1989).

${ }^{26}$ J. J. Christensen and A. J. Bray, Phys. Rev. E 58, 5364 (1998).

${ }^{27}$ L. M. Liz-Marzan, M. Giersig, and P. Mulvaney, Langmuir 12, 4329 (1996).

${ }^{28}$ Y. Lu, H. Fan, A. Stump, T. Ward, T. Rieker, and C. Brinker, Nature (London) 398, 223 (1999).

${ }^{29}$ S. Kim, W. Zhang, and T. J. Pinnavaia, Science 282, 1302 (1998).

${ }^{30}$ S. Warren, F. Disalvo, and U. Wiesner, Nature Mater. 6, 156 (2007). 